\title{
Utilization of Monte Carlo Calculations in Radiation Transport Analyses to Support the Design of the U.S. Spallation Neutron Source (SNS)
}

\author{
Jeffrey O. Johnson \\ Oak Ridge National Laboratory, P. O. Box 2008, MS-6363, Oak Ridge, Tennessee \\ 37831-6363, USA
}

\begin{abstract}
The Department of Energy (DOE) has given the Spallation Neutron Source (SNS) project approval to begin Title I design of the proposed facility to be built at Oak Ridge National Laboratory (ORNL) and construction is scheduled to commence in FY01. The SNS initially will consist of an accelerator system capable of delivering an $\sim 0.5$ microsecond pulse of $1 \mathrm{GeV}$ protons, at a $60 \mathrm{~Hz}$ frequency, with $1 \mathrm{MW}$ of beam power, into a single target station. The SNS will eventually be upgraded to a $2 \mathrm{MW}$ facility with two target stations (a $60 \mathrm{~Hz}$ station and a $10 \mathrm{~Hz}$ station). The radiation transport analysis, which includes the neutronic, shielding, activation, and safety analyses, is critical to the design of an intense high-energy accelerator facility like the proposed SNS, and the Monte Carlo method is the cornerstone of the radiation transport analyses.
\end{abstract}

\section{Introduction}

The use of neutrons in science and industry has increased continuously during the past fifty years with applications now widely used in physics, chemistry, biology, engineering, and medicine. Within this history, the relative merits of using pulsed accelerator spallation sources versus reactors for neutron sources have been debated. A consensus from the neutron scattering experiment community has finally emerged endorsing short pulse spallation sources as a high priority option for the future. To address this future need, the Department of Energy (DOE) has initiated the design of the Spallation Neutron Source (SNS) and given approval for the proposed facility to be constructed at Oak Ridge National Laboratory (ORNL). The DOE directive is to design and build a short pulse spallation source in the 1 MW power range with sufficient design flexibility that it can be upgraded and operated at a higher power at a later stage.

A diverse representation of scientific and technical disciplines are required to produce a successful spallation neutron source design, including accelerator and target station physics and engineering, remote handling, neutronics, materials, thermal hydraulics, and instrumentation. Within each of these disciplines, there are multiple layouts and designs that must be eventually integrated into a reference design. The radiation transport analysis, which includes the neutronic, shielding, activation, and safety analyses, is critical to almost all operations important to the design and construction of an intense high-energy accelerator facility like the 
proposed SNS. These analyses are fundamentally important because of the impact on conventional facility design, maintenance operations, and because the costs associated with incorporating the results of the radiation transport analysis comprise a significant part of the total facility costs. Towards the goal of producing the most optimum design, traditional concepts utilized in current facilities are being re-examined, and new concepts are being considered to meet the higher power challenge of the SNS. Since target stations are vital components of spallation neutron sources, the design of the target stations is critical to determining the overall performance of the facility as a neutron source. A brief overview of the Title I SNS design is presented in Section 2. The general philosophy adopted for the radiation transport analysis of the SNS facility is presented in Section 3, and a brief summary of the calculational methodology is discussed in Section 4.

\section{The Proposed U. S. Spallation Neutron Source}

The U. S. SNS proton accelerator system consists of a front-end ion source, linac, high-energy beam transport system, and accumulator ring. The ion source produces negative hydrogen ions that are formed into a pulsed beam and accelerated to a energy of $2.5 \mathrm{MeV}$. This beam is delivered to a large linear accelerator that accelerates the $\mathrm{H}$ - beam from $2.5 \mathrm{MeV}$ to $1 \mathrm{GeV}$. Three different types of accelerators are used in the SNS design. The first two, the drift tube linac and the coupled-cavity linac are made of copper, operate at room temperature, and accelerate the beam to about $200 \mathrm{MeV}$. The remainder of the acceleration is accomplished by super-conducting niobium cavities, cooled with liquid helium to an operating temperature of $2 \mathrm{~K}$, which accelerate the beam to $1 \mathrm{GeV}$. The proton beam is then transported to an accumulator ring structure, which bunches and intensifics the ion beam for delivery onto the mercury target to produce the pulsed neutron beams. The intense $\mathrm{H}$ - beam from the linac is wrapped into the ring through a stripper foil that strips the electrons to produce the protons that circulate in the ring. Approximately 1200 turns are accumulated, and then all the protons are extracted at once, producing a pulse of approximately 0.5 microseconds that is delivered to the target at a rate of 60 times a second.

The basic function of the SNS target system is to produce 18 to 24 lower-energy $(<1 \mathrm{eV})$, short-pulsed ( $\sim$ tens of microseconds) neutron beams optimized for neutron scattering instruments from the short pulsed ( $<1$ microsecond, $60 \mathrm{~Hz}, 17$ $\mathrm{kJ} /$ pulse), high-average power ( $1 \mathrm{MW}), 1 \mathrm{GeV}$ proton beam. The proton beam target is liquid mercury flowing inside a stainless steel container. The target is positioned within a layered iron, and high-density concrete shielding monolith approximately 12 meters in diameter. An ambient water moderator and a composite ambient water/supercritical hydrogen cryogenic moderator are positioned under the target and two supercritical hydrogen cryogenic moderators are positioned above the target. Moderator poisoning and decoupling are used to manipulate the time width and magnitude of the neutron pulses. The moderators 
also utilize ambient water pre-moderators on some sides and are surrounded by a heavy water cooled beryllium inner reflector region and lead outer reflector region. The core region, which includes the target, moderators, and beryllium/lead reflector, is contained inside a 3.5-meter diameter vessel. The target is to be installed and removed horizontally into an adjacent service cell using a target cart assembly. The target service cell is located behind the target cart assembly and measures 6 meters wide by 31 meters long by 12 meters high. Work will normally be performed via remote handling techniques behind a one-meter thick highdensity concrete wall. The other core components (e.g. moderators, reflector plug assemblies, etc.) are designed for vertical removal and are serviced in the target service cell. There are 18 to 24 neutron beam lines viewing the moderators, 9 to 12 on each side, and approximately equally spaced in angle. Each beam line has an independently operable shielding shutter controlled by the experimentalists. The beam lines are located at two levels; 9 to 12 lines directed at the ambient water and composite moderators under the target, and 9 to 12 directed at the cryogenic hydrogen moderators above the target. The shielding extends to a radius of $\sim 8$ meters at the beam line level to provide a region for the neutron beam T0 choppers.

\section{Radiation Transport Analysis Philosophy}

The radiation transport analysis of the SNS can be subdivided into four categories: (1) neutronic performance; (2) energy deposition distribution; (3) material damage and activation; and (4) shielding design and analysis. A strategy utilizing Monte Carlo calculations and coupled Monte Carlo and multi-dimensional discreteordinates calculations is being implemented to perform the radiation transport analysis. Within each of these four areas, there is an optimization procedure to follow which will yield the best design allowing for the interdependent relationships the four categories have with respect to each other and the implications associated with the overall facility design. Collaborative efforts interfacing the radiation transport analysis with the neutron and proton beam transport systems design, thermal hydraulic analysis, structural materials selection, remote handling/target maintenance requirements, and general facility layout are being implemented.

The determination of the neutronic performance involves characterizing the target station and accelerator radiation environments. Calculations are being performed to determine the neutron, proton, heavy ion, and gamma-ray flux spectra as a function of time, energy, and space for all components of the target station (target, moderators, reflectors, etc.) and accelerator (linac, ring, beam dumps, etc.). These calculations optimize (maximize or minimize) these distributions depending on the target station or accelerator component in question and desired design criteria. Within this analysis, the target/moderator/reflector configurations and material selections are being determined to yield the optimum neutron source for the experiment stations. 
The energy deposition distribution analysis is directly tied to the neutronic performance and interfaces with the thermal hydraulic analyses to determine the optimum accelerator and target station design with respect to heat transfer and fluid flow requirements. Energy deposition profiles are also being determined for the beam dumps associated with the accelerator design. These calculations are being performed for all components of the SNS rcquiring heat removal and/or subjected to thermal shock phenomena.

Material damage and activation analyses are being performed to assess facility component lifetime estimates and aid in the structural materials selection process. In particular, gas production, displacement damage, and primary knock-on atom (pka) spectra are being determined for target station and selected accelerator components. Material selections are being determined utilizing this information in conjunction with additional material issues (compatibility, machinability, costs, etc.). Activation analyses are being performed to determine facility radioactive waste streams, on-line material reprocessing requirements (mercury, liquid hydrogen, cooling water, etc.) and remote handling/maintenance requirements. Analyses are also being performed to determine background radiation levels within all parts of the facility for normal operation, shutdown, and postulated accident scenarios (single-failure/anticipated events and unlikely design basis accidents).

Shielding a spallation neutron source is more difficult than shielding a reactor neutron source because spallation neutrons have higher energies than fission neutrons. For a spallation neutron source, the highest-energy cascade neutrons approach the energy of the incident proton beam. These high-energy neutrons are extremely penetrating, and well-designed shielding is needed to prevent them from causing excessive biological dose rates. Shielding design calculations have been performed for all sections of the SNS facility. Biological shields are being designed and assessed for the proton beam transport system and associated beam dumps, the target station, the target service cell, and utility vault. Calculations are being performed for normal operation, catastrophic accident scenarios, and shutdown activation sources. Shield designs are being integrated into the overall facility design and optimized to achieve as low as reasonably achievable dose to the facility personnel, visiting experimentalists, and sensitive electronic equipment. The SNS design, consistent with other DOE accelerators, includes very rapidly-acting and highly-reliable automatic systems that cut off the beam when it is not in its desired path (or if personnel access to the tunnel is attempted during operation of the beam). The automatic beam cutoff would function to limit personnel exposures to a small number of micro-Sieverts even for worst-case beam-control accidents,

All four of these principal categories effect the environmental impact and safety design of the SNS facility. The SNS facility safety (personnel, public, environment, equipment, etc.) will be assured through reliance on both instrumentation-based active safety systems and passive shielding. 


\section{Radiation Transport Calculational Methodology}

The accuracy of the radiation transport analysis is dependent on the utilization of state-of-the-art cross-section data and computer codes. The MCNPX[1] code system has become the cornerstone computational tool used for the radiation transport analyses now that it has been officially released. MCNPX is the resulting code system from the merger, advancement, and enhancement of the LAHET[2] and MCNP4B [3] codes. The three-dimensional, multimedia, high-energy nucleonmeson transport portion (LAHET) of MCNPX is being used to obtain a detailed description of the nucleon-meson cascade. This Monte Carlo code takes into account the slowing down of charged particles via the continuous slowing-down approximation; the decay of charged pions and muons; and inelastic nucleonnucleus and charged-pion-nucleus (excluding hydrogen) collisions through the use of a multitude of high energy physics models. The MCNP4B portion of MCNPX is being used to perform the transport of the low energy $(\mathrm{E}<20 \mathrm{MeV})$ neutrons and gamma rays. For the shielding design of high power spallation targets and hadron accelerators with energies up to $2 \mathrm{GeV}$, it is beneficial to use deterministic methods for the shielding calculations instead of Monte Carlo methods because deep penetration problems and/or shield streaming problems require very high particle numbers to oblain good statistics, and high particle numbers typically lead to high computational times. Typically, in the Title I design phase of a facility like SNS, the project schedule will not accommodate Monte Carlo calculations requiring long running times. For the SNS shielding analyses, two approaches are being implemented. One approach is to couple MCNPX with the ANISN[4] onedimensional, DORT[5] two-dimensional, or TORT[6] three-dimensional discrete ordinates deterministic transport codes to analyze the deep penetration shiclding requirements. In these analyses, the newly developed Monte Carlo to discrete ordinates coupling codes (MTA, MTD, and MTT)[7], and the new HILO2k coupled 83-neutron, 22-gamma-ray cross-section library[8] are being used. HILO2k is a new high-energy neutron and photon transport cross-section library containing neutron cross sections to $2 \mathrm{GeV}$ and photon cross sections to $20 \mathrm{MeV}$. To address the shielding for shutdown activation sources, the MCNPX code provides the required input data for the isotope generation and depletion code, ORIHET95[9], which utilizes a matrix-exponential method to study the buildup and decay of activity for any system in which the nuclide production rates are known. The combination of the MCNPX(LAHET) generated high-energy spallation product source and the MCNPX(MCNP4B) low energy neutron activation source yields the radionuclide concentrations, radioactivity, and time dependent decay gamma source spectra, as a function of buildup and decay time.

\section{Conclusions}


Radiation transport analyses of the SNS design indicates that a very attractive short-pulse neutron source can be constructed using liquid mercury as the target material. Within this effort, critical path research and development activities have been identified to validate design concepts and allow future upgrades to higher power levels. A radiation transport design strategy utilizing coupled low and high : energy Monte Carlo calculations and multi-dimensional discrete ordinates calculations has been devised and implemented to characterize the neutronic performance of the proposed SNS and perform the requisite radiation transport design analyses. The radiation transport analyses are being interfaced with the neutron and proton beam transport systems design, thermal hydraulic analysis, structural materials selection, remote handling/target maintenance requirements, and general facility layout design. Lessons learned from existing facility experience are being utilized in the design. Design modifications are constantly being addressed to ascertain the impact the modifications have on the neutronic performance or radiological safety of the SNS. Future analyses will continue to refine the models to account for the details of the advanced Title I and Title II designs that effect the neutronic performance, radiological safety, or impact environmental concerns.

\section{References}

1. H. G. Hughes, et.al., "MCNPX for Neutron-Photon Transport", in International Conference on Mathematics and Computation, Reactor Physics \& Environmental Analysis in Nuclear Applications, American Nuclear Society, Madrid, Spain, September 27-30, 1999

2. R. E. Prael and H. Lichenstein, "User Guide to LCS: The LAHET Code System", LA-UR-89-3014, Los Alamos National Laboratory, (September 1989).

3. J. F. Briesmeister, Ed. "MCNP - A General Monte Carlo Code for Neutron and Photon Transport: Version 4B", LA-12625-M, Los Alamos National Laboratory (March 1997).

4. Engle, Jr., W. W., "A User's Manual for ANISN: A One-Dimensional Discrete Ordinates Transport Code with Anisotropic Scattering", Union Carbide Nuclear Division Report K-1693 (1967).

5. Rhoades, W. A. and Childs, R. L., "The DORT Two-Dimensional DiscreteOrdinates Transport Code", Nuclear Science \& Engineering 99, 1, pp. 88-89, (May 1988).

6. Rhoades, W. A. and Childs, R. L., "The TORT: A Three-Dimensional Discrete-Ordinates Neutron/Photon Transport Code", Nuclear Science \& Engineering 107, 4, pp. 397-398, (April 1991).

7. F. X. Gallmeier and R. E. Pevey, "Creation of a Set of Interface Utilities to Allow Coupled Monte Carlo/Discrete Ordinates Shielding Analysis", in 
AccApp'99 - The Third American Nuclear Society Topical Meeting on Nuclear Applications of Accelerator Technology, Long Beach CA., November $14-18,1999$.

8. R. A. Lillie and F. X. Gallmeier, "HILO2k: A Coupled Neutron-Photon Transport Cross-Section Library for Neutron Energies Up To $2000 \mathrm{MeV}$ ", To be published in AccApp'00 - The Fourth American Nuclear Society Topical Meeting on Nuclear Applications of Accelcrator Technology, Washington D.C., November 12-16, 2000.

9. Cloth, P., et al., "HERMES, A Monte Carlo Program System for Beam Material Interaction Studies," KFA Jülich, Report Jul-2203 (1988) 